\title{
Estimation of Population Mean in Chain Ratio-Type Estimator under Systematic Sampling
}

\author{
Mursala Khan' and Rajesh Singh ${ }^{2}$ \\ ${ }^{1}$ Department of Mathematics, COMSATS Institute of Information Technology, Abbottabad 22060, Pakistan \\ ${ }^{2}$ Department of Statistics, Banaras Hindu University, Varanasi 221005, India
}

Correspondence should be addressed to Mursala Khan; mursala.khan@yahoo.com

Received 7 September 2015; Accepted 11 October 2015

Academic Editor: Shein-chung Chow

Copyright (c) 2015 M. Khan and R. Singh. This is an open access article distributed under the Creative Commons Attribution License, which permits unrestricted use, distribution, and reproduction in any medium, provided the original work is properly cited.

A chain ratio-type estimator is proposed for the estimation of finite population mean under systematic sampling scheme using two auxiliary variables. The mean square error of the proposed estimator is derived up to the first order of approximation and is compared with other relevant existing estimators. To illustrate the performances of the different estimators in comparison with the usual simple estimator, we have taken a real data set from the literature of survey sampling.

\section{Introduction and Literature Review}

Incorporating the knowledge of the auxiliary variables is very important for the construction of efficient estimators for the estimation of population parameters and increasing the efficiency of the estimators in different sampling design. Using the knowledge of the auxiliary variables, several authors have proposed different estimation technique for the finite population mean of the study variable; Cochran [1], Tripathy [2], Kadilar and Cingi [3, 4], Singh et al. [5], Khan and Arunachalam [6], Lone and Tailor [7], Khan [8], Khan and Hussain [9], and Khan et al. [10] have worked on the estimation of population parameters using auxiliary information.

In the present paper, we will work on the estimation of population mean using the knowledge of the auxiliary variables under systematic sampling. Various statisticians have worked on the estimation of population mean in systematic sampling: Cochran [11], Hansen et al. [12], Robson [13], Swain [14], Singh [15], Shukla [16], Kushwaha and Singh [17], Banarasi et al. [18], R. Singh and H. P. Singh [19], Singh et al. [20], Singh and Solanki [21], Singh and Jatwa [22], Singh et al. [23, 24], Tailor et al. [25], Verma and Singh [26], and Verma et al. [27], and so forth.

Consider a finite population $U=\left\{U_{1}, U_{2}, \ldots, U_{N}\right\}$ of size $N$ units, numbered from 1 to $N$ in some order. A sample of size $n$ units is taken at random from the first $k$ units and every $k$ th subsequent unit; then, $N=n k$ where $n$ and $k$ are positive integers; thus, there will be $k$ samples (clusters) each of size $n$ and observe the study variate $y$ and auxiliary variate $x$ for each and every unit selected in the sample. Let $\left(y_{i j}, x_{i j}\right)$, for $i=$ $1,2, \ldots, k$ and $j=1,2, \ldots, n$ : denote the value of $j$ th unit in the $i$ th sample. Then, the systematic sample means are defined as follows: $\bar{y}_{s y}=t_{0}=(1 / n) \sum_{j=1}^{n} y_{i j}$ and $\bar{x}_{s y}=(1 / n) \sum_{j=1}^{n} x_{i j}$ are the unbiased estimators of the population means $\bar{Y}=$ $(1 / N) \sum_{j=1}^{N} y_{i j}$ and $\bar{X}=(1 / N) \sum_{j=1}^{N} x_{i j}$ of $y$ and $x$, respectively. Let $S_{y}^{2}=(1 /(N-1)) \sum_{i=1}^{k} \sum_{j=1}^{n}\left(y_{i j}-\bar{Y}\right)^{2}, S_{x}^{2}=(1 /(N-$ 1)) $\sum_{i=1}^{k} \sum_{j=1}^{n}\left(x_{i j}-\bar{X}\right)^{2}$, and $S_{z}^{2}=(1 /(N-1)) \sum_{i=1}^{k} \sum_{j=1}^{n}\left(z_{i j}-\right.$ $\bar{Z})^{2}$ be the population variances of the study variable and the auxiliary variables, respectively, with the corresponding population covariance's $S_{y x}=(1 /(N-1)) \sum_{i=1}^{k} \sum_{j=1}^{n}\left(x_{i j}-\right.$ $\bar{X})\left(y_{i j}-\bar{Y}\right), S_{y z}=(1 /(N-1)) \sum_{i=1}^{k} \sum_{j=1}^{n}\left(y_{i j}-\bar{Y}\right)\left(z_{i j}-\bar{Z}\right)$, and $S_{x z}=(1 /(N-1)) \sum_{i=1}^{k} \sum_{j=1}^{n}\left(x_{i j}-\bar{X}\right)\left(z_{i j}-\bar{Z}\right)$ among the three variables $y, x$, and $z$, respectively. Also $C_{y}^{2}, C_{x}^{2}$, and $C_{z}^{2}$ are the known population coefficients of variation of the study variable and the auxiliary variables, respectively.

To obtain the properties of the estimators up to first order of approximation, we use the following errors terms: 
$e_{0}=\left(\bar{y}_{s y s}-\bar{Y}\right) / \bar{Y}, e_{1}=\left(\bar{x}_{s y s}-\bar{X}\right) / \bar{X}$, and $e_{2}=\left(\bar{z}_{s y s}-\bar{Z}\right) / \bar{Z}$, such that $E\left(e_{i}\right)=0$, for $i=0,1$, and 2 .

The first order of approximation of the above errors terms is given by

$$
\begin{gathered}
E\left(e_{0}^{2}\right)=\theta \rho_{y}^{*} C_{y}^{2}, \\
E\left(e_{1}^{2}\right)=\theta \rho_{x}^{*} C_{x}^{2}, \\
E\left(e_{2}^{2}\right)=\theta \rho_{z}^{*} C_{z}^{2}, \\
E\left(e_{0} e_{1}\right)=\theta k C_{x}^{2} \sqrt{\rho_{y}^{*} \rho_{x}^{*}}, \\
E\left(e_{0} e_{2}\right)=\theta k^{*} C_{z}^{2} \sqrt{\rho_{y}^{*} \rho_{z}^{*}}, \\
E\left(e_{1} e_{2}\right)=\theta k^{* *} C_{z}^{2} \sqrt{\rho_{x}^{*} \rho_{z}^{*}},
\end{gathered}
$$

where

$$
\begin{aligned}
\lambda & =\left(\frac{N-1}{n N}\right), \\
\rho_{y x} & =\frac{S_{y x}}{S_{y} S_{x}}, \\
\rho_{y z} & =\frac{S_{y z}}{S_{y} S_{z}}, \\
\rho_{x z} & =\frac{S_{x z}}{S_{x} S_{z}}, \\
k & =\frac{\rho_{y x} C_{y}}{C_{x}}, \\
k^{*} & =\frac{\rho_{y z} C_{y}}{C_{z}}, \\
\rho_{y}^{*} & =\left\{1+(n-1) \rho_{y}\right\}, \\
\rho_{x}^{*} & =\left\{1+(n-1) \rho_{x}\right\}, \\
\rho_{z}^{*} & =\left\{1+(n-1) \rho_{z}\right\}, \\
\rho^{* *} & =\frac{\rho_{y}^{*}}{\rho_{x}^{*}}, \\
\rho_{2}^{* *} & =\frac{\rho_{y}^{*}}{\rho_{z}^{*}}, \\
\rho_{1}^{* *} & =\frac{\rho_{x}^{*}}{\rho_{z}^{*}},
\end{aligned}
$$

where $\rho_{y}, \rho_{x}$, and $\rho_{z}$ are the intraclass correlation among the pair of units for the variables $y, x$, and $z$, respectively.

The variance of the usual unbiased estimator for population mean is

$$
\operatorname{Var}\left(t_{0}\right)=\lambda \bar{Y}^{2} \rho_{y}^{*} C_{y}^{2}
$$

The classical ratio and product estimators for finite population mean suggested by Swain [14] and Shukla [16] are given by

$$
\begin{aligned}
& t_{1}=\bar{y}_{s y}\left(\frac{\bar{X}}{\bar{x}_{s y}}\right), \\
& t_{2}=\bar{y}_{s y} \exp \left(\frac{\bar{z}_{s y}}{\bar{Z}}\right) .
\end{aligned}
$$

The mean square errors of the estimators, to the first order of approximation, are given as follows:

$$
\begin{aligned}
& \operatorname{MSE}\left(t_{1}\right)=\lambda \bar{Y}^{2}\left[\rho_{y}^{*} C_{y}^{2}+\rho_{x}^{*} C_{x}^{2}\left(1-2 k \sqrt{\rho^{* *}}\right)\right], \\
& \operatorname{MSE}\left(t_{2}\right)=\lambda \bar{Y}^{2}\left[\rho_{y}^{*} C_{y}^{2}+\rho_{z}^{*} C_{z}^{2}\left(1+2 k^{*} \sqrt{\rho_{2}^{* *}}\right)\right] .
\end{aligned}
$$

The usual regression estimator, using single auxiliary variable and its variance, is given as follows:

$$
\begin{aligned}
t_{3} & =\bar{y}_{s y}+b\left(\bar{X}-\bar{x}_{s y}\right), \\
\operatorname{MSE}\left(t_{3}\right) & =\lambda \rho_{y}^{*} S_{y}^{2}\left[1-\rho_{y x}^{2}\right] .
\end{aligned}
$$

Utilizing the known knowledge of the auxiliary variable, Singh et al. [20] suggested the following ratio and product type exponential estimators:

$$
\begin{aligned}
& t_{4}=\bar{y}_{s y} \exp \left(\frac{\bar{X}-\bar{x}_{s y}}{\bar{X}+\bar{x}_{s y}}\right), \\
& t_{5}=\bar{y}_{s y} \exp \left(\frac{\bar{x}_{s y}-\bar{X}}{\bar{x}_{s y}+\bar{X}}\right) .
\end{aligned}
$$

The mean square errors of the estimators up to first order of approximation are given by

$$
\begin{aligned}
& \operatorname{MSE}\left(t_{4}\right)=\lambda \bar{Y}^{2}\left[\rho_{y}^{*} C_{y}^{2}+\frac{\rho_{x}^{*} C_{x}^{2}}{4}\left(1-4 k \sqrt{\rho^{* *}}\right)\right], \\
& \operatorname{MSE}\left(t_{5}\right)=\lambda \bar{Y}^{2}\left[\rho_{y}^{*} C_{y}^{2}+\frac{\rho_{x}^{*} C_{x}^{2}}{4}\left(1+4 k \sqrt{\rho^{* *}}\right)\right] .
\end{aligned}
$$

After that, Tailor et al. [25] define the following ratio-cumproduct estimator for the population mean $\bar{Y}$ :

$$
t_{6}=\bar{y}_{s y}\left(\frac{\bar{X}}{\overline{\bar{x}}_{s y}}\right)\left(\frac{\bar{z}_{s y}}{\bar{Z}}\right) .
$$

The mean square error of the estimator $t_{6}$, up to first order of approximation, is given by

$$
\begin{gathered}
\operatorname{MSE}\left(t_{6}\right)=\lambda \bar{Y}^{2}\left[\rho_{y}^{*} C_{y}^{2}+\rho_{x}^{*} C_{x}^{2}\left(1-2 k \sqrt{\rho^{* *}}\right)\right. \\
\left.+\rho_{z}^{*} C_{z}^{2}\left(1-2 k^{* *} \sqrt{\rho_{1}^{* *}}\right)+2 k^{*} C_{z}^{2} \sqrt{\rho_{y}^{*} \rho_{z}^{*}}\right] .
\end{gathered}
$$




\section{Proposed Estimator}

In this section, we have proposed the following regression in ratio-cum-product type estimator for the unknown population mean under systematic sampling:

$$
\begin{gathered}
t_{m}=\bar{y}_{s y}\left(\frac{\bar{X}}{\bar{X}+b_{y x}\left(\bar{x}_{s y}-\bar{X}\right)}\right)^{\delta_{1}} \\
\cdot\left(\frac{\bar{Z}+b_{y z}\left(\bar{z}_{s y}-\bar{Z}\right)}{\bar{Z}}\right)^{\delta_{2}},
\end{gathered}
$$

where $\delta_{1}$ and $\delta_{2}$ are the unknown constants, whose values are to be found for the minimum mean square error.

The mean square error (MSE) of the estimator up to first order of approximation is

$$
\begin{aligned}
& \operatorname{MSE}\left(t_{m}\right)=\lambda \bar{Y}^{2}\left[\rho_{y}^{*} C_{y}^{2}+\delta_{1}^{2} \beta_{y x}^{2} \rho_{x}^{*} C_{x}^{2}+\delta_{2}^{2} \beta_{y z}^{2} \rho_{z}^{*} C_{z}^{2}\right. \\
& -2 \delta_{1} \beta_{y x} k C_{x}^{2} \sqrt{\rho_{y}^{*} \rho_{x}^{*}}+2 \delta_{2} \beta_{y z} k^{*} C_{z}^{2} \sqrt{\rho_{y}^{*} \rho_{z}^{*}} \\
& \left.-2 \delta_{1} \delta_{2} \beta_{y x} \beta_{y z} k^{* *} C_{z}^{2} \sqrt{\rho_{x}^{*} \rho_{z}^{*}}\right] .
\end{aligned}
$$

On differentiating (15), with respect to $\delta_{1}$ and $\delta_{2}$, we obtain the minimum mean squared error of the estimator $t_{m}$, which is given by

$$
\begin{aligned}
& \operatorname{MSE}\left(t_{m}\right) \\
& =\lambda \bar{Y}^{2} \rho_{y}^{*}\left[C_{y}^{2}-k_{x}^{2 *} C_{z}^{2}-\frac{\left(k^{*} k^{* *} C_{z}^{2}-k C_{x}^{2}\right)^{2}}{\left(C_{x}^{2}-k^{2 * *} C_{z}^{2}\right)}\right],
\end{aligned}
$$

where the optimum values are $\delta_{1}=\sqrt{\rho_{y}^{*}}\left(k C_{x}^{2}-k^{*} k^{* *} C_{z}^{2}\right) /$ $\beta_{y x} \sqrt{\rho_{x}^{*}}\left(C_{x}^{2}-k^{2 * *} C_{z}^{2}\right)$ and $\delta_{2}=\left(\sqrt{\rho_{y}^{*}} / \beta_{y z} \sqrt{\rho_{z}^{*}}\right)\left\{k^{* *}\left(k C_{x}^{2}-\right.\right.$ $\left.\left.k^{*} k^{* *} C_{z}^{2}\right) /\left(C_{x}^{2}-k^{2 * *} C_{z}^{2}\right)-k^{*}\right\}$.

\section{Comparison}

In this section, we have compared the MSE of the proposed estimator with the MSEs of simple estimator, Swain [14] estimator, Shukla [16] estimator, Singh et al. [20] estimators, and Tailor et al. [25] estimator and found some theoretical conditions under which the proposed estimator will always perform better:

(i) By (16) and (3), $\operatorname{MSE}\left(t_{m}\right) \leq \operatorname{MSE}\left(t_{0}\right)$ if

$$
\left[k_{x}^{2 *} C_{z}^{2}+\frac{\left(k^{*} k^{* *} C_{z}^{2}-k C_{x}^{2}\right)^{2}}{\left(C_{x}^{2}-k^{2 * *} C_{z}^{2}\right)}\right] \geq 0 .
$$

(ii) By (16) and (5), $\operatorname{MSE}\left(t_{m}\right) \leq \operatorname{MSE}\left(t_{1}\right)$ if

$$
\begin{aligned}
& {\left[\rho_{y}^{*}\left\{k_{x}^{2 *} C_{z}^{2}+\frac{\left(k^{*} k^{* *} C_{z}^{2}-k C_{x}^{2}\right)^{2}}{\left(C_{x}^{2}-k^{2 * *} C_{z}^{2}\right)}\right\}\right.} \\
& \left.+\rho_{x}^{*} C_{x}^{2}\left(1-2 k \sqrt{\rho^{* *}}\right)\right] \geq 0 .
\end{aligned}
$$

(iii) By (16) and (6), $\operatorname{MSE}\left(t_{m}\right) \leq \operatorname{MSE}\left(t_{2}\right)$ if

$$
\begin{aligned}
& {\left[\rho_{y}^{*}\left\{k_{x}^{2 *} C_{z}^{2}+\frac{\left(k^{*} k^{* *} C_{z}^{2}-k C_{x}^{2}\right)^{2}}{\left(C_{x}^{2}-k^{2 * *} C_{z}^{2}\right)}\right\}\right.} \\
& \left.+\rho_{z}^{*} C_{z}^{2}\left(1+2 k^{*} \sqrt{\rho_{2}^{* *}}\right)\right] \geq 0 .
\end{aligned}
$$

(iv) By (16) and (8), $\operatorname{MSE}\left(t_{m}\right) \leq \operatorname{MSE}\left(t_{3}\right)$ if

$$
\left[k_{x}^{2 *} C_{z}^{2}-C_{y}^{2} \rho_{y x}^{2}+\frac{\left(k^{*} k^{* *} C_{z}^{2}-k C_{x}^{2}\right)^{2}}{\left(C_{x}^{2}-k^{2 * *} C_{z}^{2}\right)}\right] \geq 0 .
$$

(v) By (16) and (10), $\operatorname{MSE}\left(t_{m}\right) \leq \operatorname{MSE}\left(t_{4}\right)$ if

$$
\begin{gathered}
{\left[\rho_{y}^{*}\left\{k_{x}^{2 *} C_{z}^{2}+\frac{\left(k^{*} k^{* *} C_{z}^{2}-k C_{x}^{2}\right)^{2}}{\left(C_{x}^{2}-k^{2 * *} C_{z}^{2}\right)}\right\}\right.} \\
\left.+\frac{\rho_{x}^{*} C_{x}^{2}}{4}\left(1-4 k \sqrt{\rho^{* *}}\right)\right] \geq 0 .
\end{gathered}
$$

(vi) By (16) and (11), $\operatorname{MSE}\left(t_{m}\right) \leq \operatorname{MSE}\left(t_{5}\right)$ if

$$
\begin{aligned}
& {\left[\frac{\rho_{x}^{*} C_{x}^{2}}{4}\left(1+4 k \sqrt{\rho^{* *}}\right)\right.} \\
& \left.+\rho_{y}^{*}\left\{k_{x}^{2 *} C_{z}^{2}+\frac{\left(k^{*} k^{* *} C_{z}^{2}-k C_{x}^{2}\right)^{2}}{\left(C_{x}^{2}-k^{2 * *} C_{z}^{2}\right)}\right\}\right] \geq 0 .
\end{aligned}
$$

(vii) By (16) and (13), $\operatorname{MSE}\left(t_{m}\right) \leq \operatorname{MSE}\left(t_{6}\right)$ if

$$
\begin{aligned}
& {\left[\rho_{x}^{*} C_{x}^{2}\left(1-2 k \sqrt{\rho^{* *}}\right)+\rho_{z}^{*} C_{z}^{2}\left(1-2 k^{* *} \sqrt{\rho_{1}^{* *}}\right)\right.} \\
& +2 k^{*} C_{z}^{2} \sqrt{\rho_{y}^{*} \rho_{z}^{*}} \\
& \left.+\rho_{y}^{*}\left\{k_{x}^{2 *} C_{z}^{2}+\frac{\left(k^{*} k^{* *} C_{z}^{2}-k C_{x}^{2}\right)^{2}}{\left(C_{x}^{2}-k^{2 * *} C_{z}^{2}\right)}\right\}\right] \geq 0 .
\end{aligned}
$$


TABLE 1: The percent relative efficiency of different estimators with respect to $t_{0}$.

\begin{tabular}{lcc}
\hline & Population & \\
\hline Estimator & $\operatorname{MSE}\left(t_{\alpha}\right)$ & $\operatorname{PRE}\left(t_{\alpha}, t_{0}\right)$ \\
\hline$t_{0}$ & 1455.08 & 100.00 \\
$t_{1}$ & 373.32 & 389.62 \\
$t_{2}$ & 768.06 & 189.45 \\
$t_{3}$ & 45.52 & 3196.57 \\
$t_{4}$ & 820.09 & 177.43 \\
$t_{5}$ & 1044.42 & 139.32 \\
$t_{6}$ & 187.08 & 777.79 \\
$t_{m}$ & 22.74 & 6400.00 \\
\hline
\end{tabular}

\section{Numerical Comparison}

For comparing the theoretical efficiency conditions of the different estimators numerically, we have used the following real data set.

Population 1 (source: Tailor et al. [25]). Consider

$$
\begin{aligned}
N & =15, \\
n & =3, \\
\bar{X} & =44.47, \\
\bar{Y} & =80, \\
\bar{Z} & =48.40, \\
C_{y} & =0.56, \\
C_{x} & =0.28, \\
C_{z} & =0.43, \\
S_{y}^{2} & =2000, \\
S_{x}^{2} & =149.55, \\
S_{z}^{2} & =427.83, \\
S_{y x} & =538.57, \\
S_{y z} & =-902.86, \\
S_{x z} & =-241.06, \\
\rho_{y x} & =0.9848, \\
\rho_{y z} & =-0.9760, \\
\rho_{x z} & =-0.9530, \\
\rho_{y} & =0.6652, \\
\rho_{x} & =0.707, \\
\rho_{z} & =0.5487 .
\end{aligned}
$$

For the percent relative efficiencies (PREs) of the estimator, we use the following formula and the results are shown in Table 1:

$\operatorname{PRE}\left(t_{\alpha}, t_{0}\right)=\operatorname{MSE}\left(t_{0}\right) / \operatorname{MSE}\left(t_{\alpha}\right) \times 100$, for $\alpha=0,1,2$, $3,4,5,6$, and $m$.

\section{Conclusion}

A chain ratio-type estimator is proposed under double sampling scheme using two auxiliary variables, and the properties of the proposed estimator are derived up to first order of approximations. Both theoretically and empirically, it has been shown that the recommended estimator performed better than the other competing estimators in terms of higher percent relative efficiency. Hence, looking on the dominance nature of the proposed estimator may be suggested for its practical applications.

\section{Conflict of Interests}

The authors declare that there is no conflict of interests regarding the publication of this paper.

\section{Acknowledgment}

The authors are thankful to the anonymous learned referees for their valuable suggestions regarding the improvement of the paper.

\section{References}

[1] W. G. Cochran, Sampling Techniques, John Wiley \& Sons, New York, NY, USA, 1977.

[2] T. P. Tripathy, "A general class of estimators of population ratio," Sankhya Series C, vol. 42, pp. 45-63, 1980.

[3] C. Kadilar and H. Cingi, "Ratio estimators in simple random sampling," Applied Mathematics and Computation, vol. 151, no. 3, pp. 893-902, 2004.

[4] C. Kadilar and H. Cingi, "Improvement in estimating the population mean in simple random sampling," Applied Mathematics Letters, vol. 19, no. 1, pp. 75-79, 2006.

[5] R. Singh, P. Chauhan, N. Sawan, and F. Smarandache, "Improved exponential estimator for population variance using two auxiliary variables," Italian Journal of Pure and Applied Mathematics, vol. 28, pp. 101-108, 2011.

[6] M. Khan and A. Arunachalam, "Estimation of population variance using the knowledge of kurtosis of an auxiliary variable under simple random sampling," International Journal of Applied Science and Mathematics, vol. 1, no. 2, pp. 63-67, 2014.

[7] H. A. Lone and R. Tailor, "Dual to separate product type exponential estimator in sample surveys," Journal of Statistics Applications \& Probability Letters, vol. 2, no. 2, pp. 89-96, 2015.

[8] M. Khan, "Improvement in estimating the finite population mean under maximum and minimum values in double sampling scheme," Journal of Statistics Applications \& Probability Letters, vol. 2, no. 2, pp. 1-7, 2015.

[9] M. Khan and S. Hussain, "An improved class of ratio-type estimators for finite population mean under maximum and minimum values," Science International, vol. 27, no. 2, pp. 11351138, 2015.

[10] M. Khan, S. Ullah, A. Y. Al-Hossain, and N. Bashir, "Improved ratio-type estimators using maximum and minimum values under simple random sampling scheme," Hacettepe Journal of Mathematics and Statistics, vol. 44, no. 4, pp. 923-931, 2015. 
[11] W. G. Cochran, "The estimation of the yields of cereal experiments by sampling for the ratio of grain to total produce," The Journal of Agricultural Science, vol. 30, no. 2, pp. 262-275, 1940.

[12] M. H. Hansen, W. N. Hurwitz, and M. Gurney, "Problems and methods of the sample survey of business," The Journal of the American Statistical Association, vol. 41, no. 234, pp. 173-189, 1946.

[13] D. S. Robson, "Application of multivariate polykays to the theory of unbiased ratio-type estimation," The Journal of the American Statistical Association, vol. 59, pp. 1225-1226, 1957.

[14] A. K. P. C. Swain, "The use of systematic sampling ratio estimate," Journal of Indian Statistical Association, vol. 2, pp. 160-164, 1964.

[15] M. P. Singh, "Ratio cum product method of estimation," Metrika, vol. 12, no. 1, pp. 34-42, 1967.

[16] N. D. Shukla, "Systematic sampling and product method of estimation," in Proceeding of All India Seminar on Demography and Statistics, BHU, Varanasi, India, 1971.

[17] K. S. Kushwaha and H. P. Singh, "Class of almost unbiased ratio and product estimators in systematic sampling," Journal of the Indian Society of Agricultural Statistics, vol. 41, no. 2, pp. 193205, 1989.

[18] Banarasi, S. N. S. Kushwaha, and K. S. Kushwaha, "A class of ratio, product and difference (RPD) estimators in systematic sampling," Microelectronics Reliability, vol. 33, no. 4, pp. 455457, 1993.

[19] R. Singh and H. P. Singh, "Almost unbiased ratio and product type estimators in systematic sampling," Questiio, vol. 22, no. 3, pp. 403-416, 1998.

[20] H. P. Singh, R. Tailor, and N. K. Jatwa, "Modified ratio and product estimators for population mean in systematic sampling," Journal of Modern Applied Statistical Methods, vol. 10, no. 2, pp. 424-435, 2011.

[21] H. P. Singh and R. S. Solanki, "An efficient class of estimators for the population mean using auxiliary information in systematic sampling," Journal of Statistical Theory and Practice, vol. 6, no. 2, pp. 274-285, 2012.

[22] H. P. Singh and N. K. Jatwa, "A class of exponential type estimators in systematic sampling," Economic Quality Control, vol. 27, no. 2, pp. 195-208, 2012.

[23] R. Singh, S. Malik, and V. K. Singh, "An improved estimator in systematic sampling," Journal of Scientific Research, vol. 56, pp. 177-182, 2012.

[24] R. Singh, S. Malik, M. K. Chaudhary, H. Verma, and A. A. Adewara, "A general family of ratio-type estimators in systematic sampling," Journal of Reliability and Statistical Studies, vol. 5, no. 1, pp. 73-82, 2012.

[25] T. Tailor, N. K. Jatwa, and H. P. Singh, "A ratio-cum-product estimator of finite population mean in systematic sampling," Statistics in Transition, vol. 14, no. 3, pp. 391-398, 2013.

[26] H. K. Verma and R. Singh, "A family of efficient estimator in circular systematic sampling," Journal of Advanced Computing, vol. 3, no. 2, pp. 56-68, 2014.

[27] H. K. Verma, R. D. Singh, and R. Singh, "Some improved estimators in systematic sampling under non-response," National Academy Science Letters, vol. 37, no. 1, pp. 91-95, 2014. 


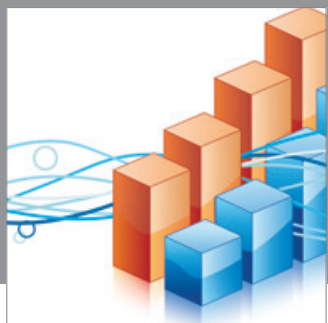

Advances in

Operations Research

mansans

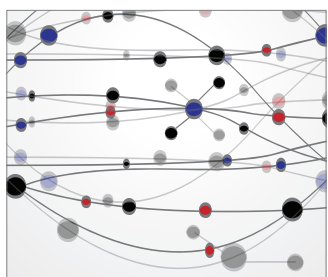

The Scientific World Journal
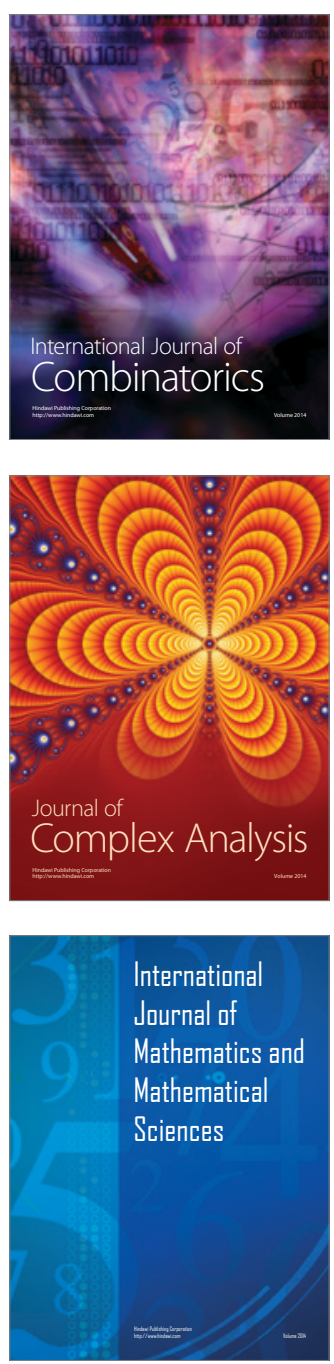
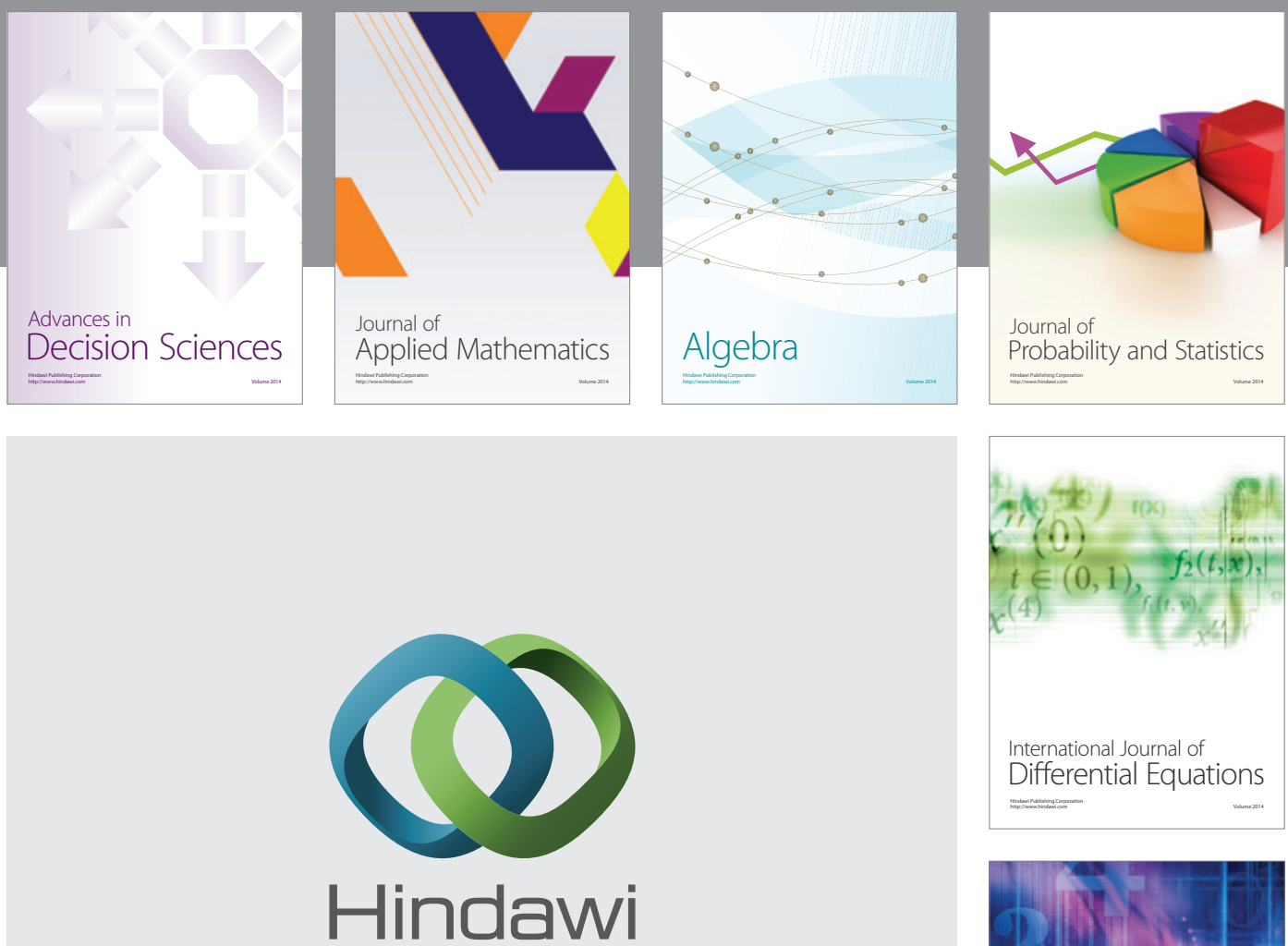

Submit your manuscripts at http://www.hindawi.com
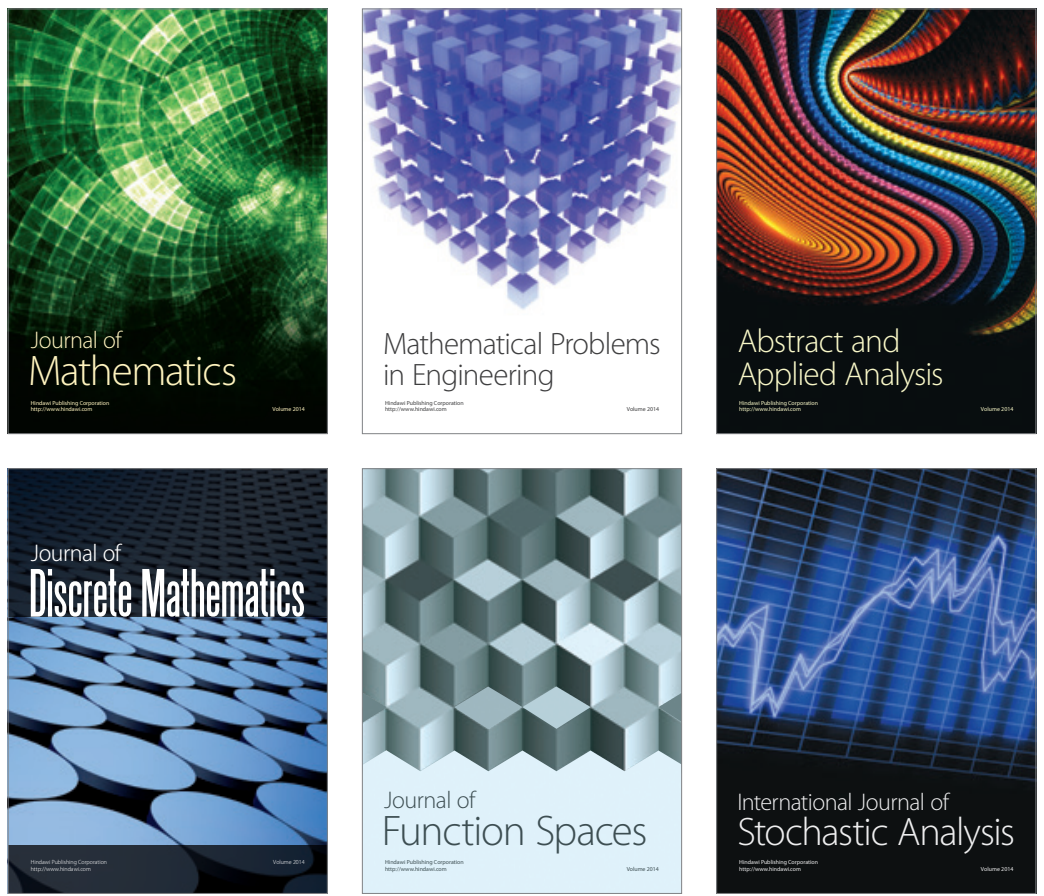

Journal of

Function Spaces

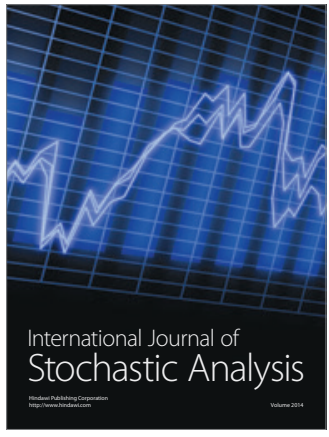

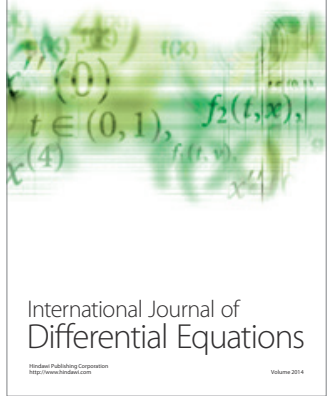
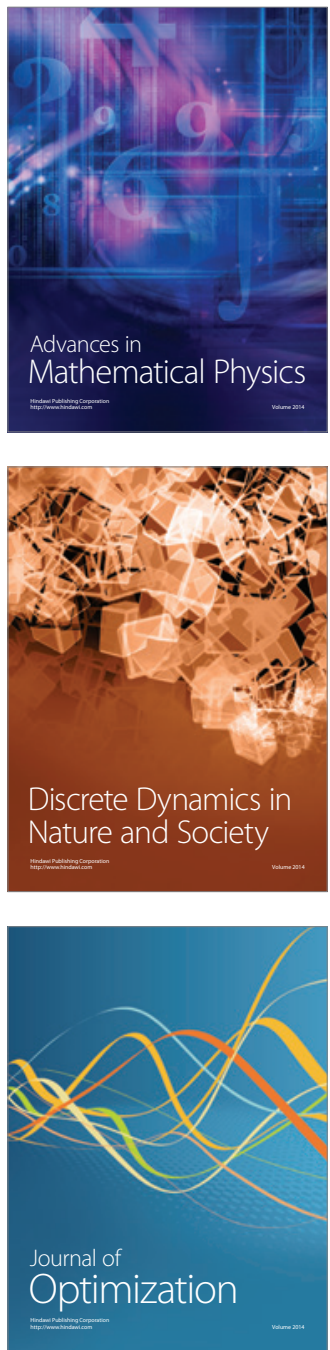